\title{
Acquisition-Related Limitations in MRI Based Morphometry
}

\author{
Arne Littmann ${ }^{1,2}$, Jens Guehring ${ }^{1}$, Christian Buechel ${ }^{3}$, \\ and Hans-Siegfried Stiehl ${ }^{2}$ \\ ${ }^{1}$ Siemens Medical Solutions, Magnetic Resonance, Erlangen, Germany \\ ${ }^{2}$ Department of Informatics, University of Hamburg, Germany \\ ${ }^{3}$ NeuroImage Nord, University Hospital Eppendorf, Hamburg, Germany \\ arne.littmannasiemens.com
}

\begin{abstract}
Although significant effort has been spent over the past decades to develop innovative image processing algorithms and to improve existing methods in terms of precision, reproducibility and computational efficiency, relatively few research was undertaken to find out to what extent the validity of results obtained with these methods is limited by inherent imperfections of the input images. This observation is especially true for MRI based morphometry, which aims at precise and highly reproducible determination of geometrical properties of anatomical structures despite the fact that MR images are geometrically distorted. We here present (a) a method for characterization of sitespecific geometrical distortions and (b) the results of a long term study designed to find out how precisely geometrical properties and morphological changes of brain structures can, in principle, be detected in images acquired with MRI scanners. Due to the long-term character of our study, our findings include effects resulting from limited hardware stability as well as from variations in patient positioning. Our results show that these effects can be strong enough to substantially confound MRI studies of small morphological changes.
\end{abstract}

\section{Introduction}

Over the last decade MRI based morphometry has experienced a remarkable gain in attention in medical research, one of the most prominent areas of application being the detection of statistically significant morphological differences in the brain between a population known to be affected by a certain disease and supposedly healthy controls. Recently, serial studies examining the progress of diseases over time have attracted attention, and it can be expected that in the future single subject applications of morphometry will become more and more important in the context of computer aided detection and staging of diseases.

Even though there exists a great variety of methods that allow for these demanding morphological analyses [1-3], few research was undertaken to find out to what extent the validity of results obtained with these methods is limited by inherent imperfections in the data acquisition process. For a profound characterization of small phenomena like beginning gray matter (GM) atrophy in early Alzheimer's disease, how- 
ever, it is inevitable to exactly know to what extent the results obtained with state-ofthe-art morphometric methods might be confounded by these limitations.

Only very little information concerning these effects could be found in the literature: Jovicich et al. either characterize distortions by the change and variance of a phantom's total diameter [4] or evaluate the influence of gradient nonlinearity correction on hippocampus volumetry in single session repositioning experiments [5]. To our knowledge no publications are available concerning the precise quantification of the crucial long-term reproducibility of geometrical distortions on a local basis. We therefore developed a new method for characterization of site-specific geometrical distortions and used it for quantitative analyses of the previously mentioned limitations in a long-term study carried out on state-of-the-art MR scanners. Our evaluation method is capable of determining positional and volumetric deviations on a dense 3D grid both in an absolute and in a relative way. We additionally compared our findings to results obtained from the analysis of head images of a healthy volunteer acquired in the same imaging sessions with identical protocols.

\subsection{Sources of Geometrical Distortion}

A detailed description of the different sources of geometrical distortions in MRI is beyond the scope of this paper, which is why we restrict our delineation of these error sources to a basic level. More information on this topic can be found in [6]. In short, there are four main sources of geometrical distortions in MRI:

- Magnetic susceptibility differs between different materials which is why their larmor-frequency varies slightly. This leads to geometrical distortions mainly in frequency-encoding direction whose magnitude in image space is also depending on the imaging protocol's bandwidth.

- Static field inhomogeneities cause distortions in the same way as differences in magnetic susceptibility. Typically these errors are compensated for the most part by shimming.

- Gradient coil nonlinearities cause distortions in all directions. Since gradient coils are designed to minimize these effects around the magnet's isocenter, nonlinearities are stronger in outer regions.

- Eddy currents are induced in conductive material within the scanner every time a gradient is changed in the course of the scanning process. As eddy currents cause dynamic magnetic fields, geometrical distortions arise. Quite obviously, errors caused by eddy currents are dependent on the actual imaging sequence and its parameterization.

Whereas static field inhomogeneities, gradient nonlinearities and eddy currents are independent of the object being scanned (as long as it is not conductive), and thus, stationary in the coordinate system of the magnet, magnetic susceptibility artifacts are a characteristic of the object and can be thought of as approximately stationary in the object's coordinate system. This distinction is important to take into consideration when analyzing relative distortions between two (or more) scans caused by slight differences of the object's position in the scanner. 


\section{Methods}

\subsection{Data Acquisition}

Based on our literature research, MPRAGE was identified as the most commonly used sequence in brain morphometry today. In cooperation with sequence developers at Siemens Medical Solutions, Magnetic Resonance, Erlangen, the following protocol was defined for the Avanto 1,5T and the Trio 3T systems: TR $2300 \mathrm{~ms}$, TI $1100 \mathrm{ms,}$ TE $3.93 \mathrm{~ms}$, flip angle $12^{\circ}$, matrix 224 x 256, bandwidth $130 \mathrm{~Hz} /$ pixel, asymmetric echoes allowed. All datasets were acquired in sagittal orientation and with an isotropic resolution of $1.0 \mathrm{~mm}$.

Using this protocol, a phantom and a healthy volunteer $(\mathrm{m}, 28 \mathrm{y})$ were regularly scanned over 6 months alternating between an Avanto and a Trio. The phantom is cylindrically shaped (diameter $250 \mathrm{~mm}$ ) with an integrated 3D grid of small spherical holes (4 mm radius) each having a distance of $20 \mathrm{~mm}$ to its nearest neighbor in $\mathrm{x}$ - and $\mathrm{y}$-, and $19 \mathrm{~mm}$ in z-direction (979 spheres in total). These holes are connected in $\mathrm{z}$ direction by cylindrical bars ( $2 \mathrm{~mm}$ radius), and the complete system of cavities is filled with fluid.
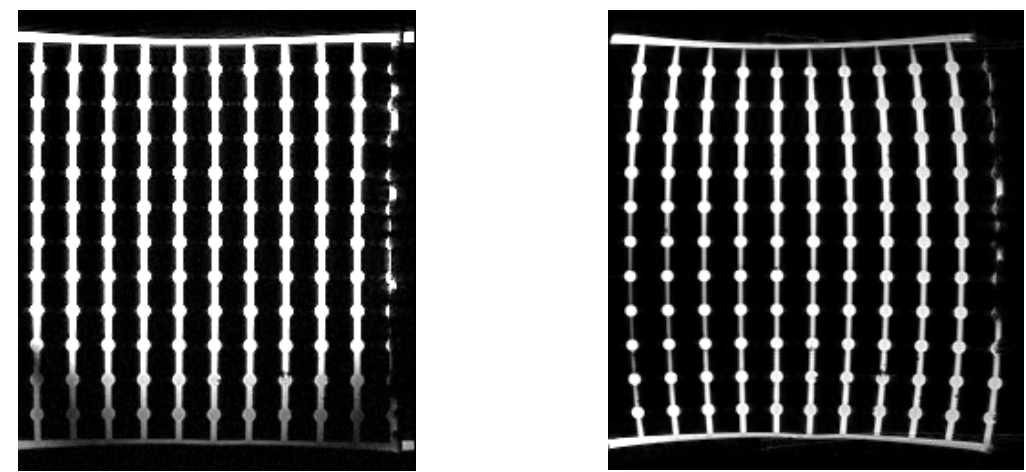

Fig. 1. Two slices of a phantom dataset acquired with the Avanto (left) and Trio (right). The interruptions in the bright bars are caused by inclusions of air.

\subsection{Phantom Data Evaluation}

The method for evaluating each of the phantom datasets is a two-stage procedure: First, the ellipsoids' center points are determined, and then this set of center points is compared to a reference phantom model $G 0$ obtained from the analysis of a highresolution CT-scan ( $0.5 \mathrm{~mm}$ isotropic resolution).

The identification of the ellipsoids, again, can be divided into two passes: In a 2D operation, they are first separated from the interconnecting bars by eliminating all those pixels that belong to an object whose bounding box is smaller than a userdefined minimum. With the subsequent connected component analysis the remaining $2 \mathrm{D}$ objects are combined into $3 \mathrm{D}$ blobs again: 


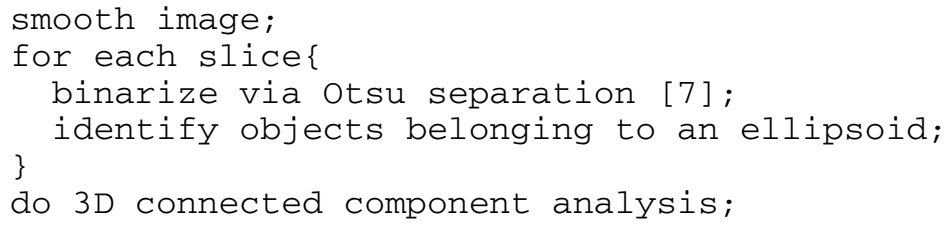

These blobs are then filtered by position (to eliminate irregular ellipsoids occurring as a result of wrap-around artifacts), and by shape (to eliminate degenerated objects resulting from air inclusions).

The second pass consists of a computationally more demanding determination of the remaining ellipsoids' center points. Its principle idea is to determine the pointsymmetry center for each ellipsoid in the original image. To ease the optimization strategy, candidate center points need to be located on the interconnecting bar's center line, which is determined via regression analysis. As a metric for symmetry the negative summed squared difference between each voxel and its mirrored counterpart (resulting from $3^{\text {rd }}$ order B-spline interpolation) was used:

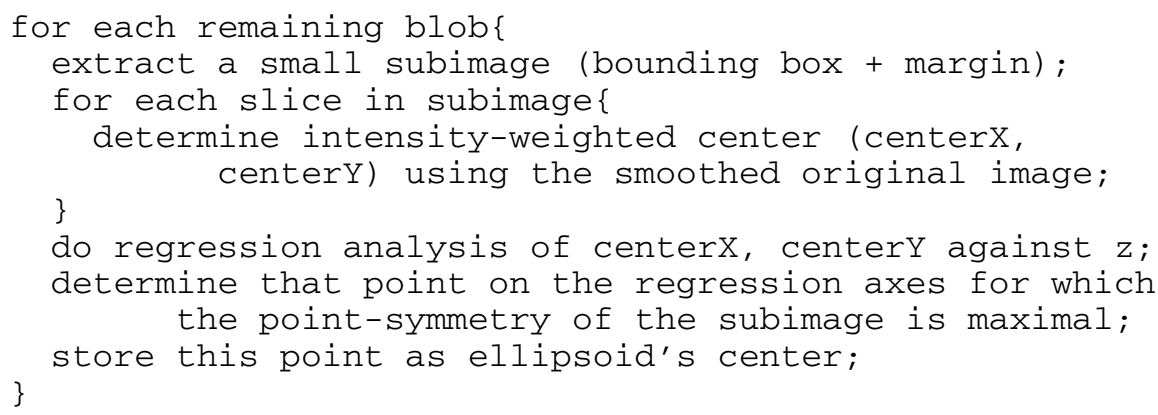

The resulting point set $G 1$ is then compared to the previously mentioned reference phantom model $G 0$ :

- The positional comparison is done straightforward and merely consists of a calculation of the absolute deviation in position for each sphere compared to its position according to the reference model $G 0$.

- For volumetric analysis $G 1$ is subdivided into polyhedral cells by iteratively grouping eight adjacent center points together in order to form one cell, the shape of which can be intuitively described as a "degenerated cuboid". For determination of the detected cells' volumes, the polyhedrons are further divided into tetrahedrons, the volumes of which can be easily calculated; finally, each cell's volume is compared to the volume of the corresponding cell in $G 0$.

\subsection{Head Image Evaluation}

Since this project's main focus was the precise quantification of limitations in serial MRI based morphometry resulting from data acquisition alone, it was put more emphasis on the phantom experiments than on the head image evaluations, which were expected to be further confounded by movements during scan, morphological changes due to differences in the volunteer's water balance and by a presumably less precise 
segmentation procedure. The head image evaluation therefore was merely done for verification purposes. It was almost completely carried out with SPM2 [3].

After correcting each dataset for intensity inhomogeneities, a template image was created for each data series (one template for each scanner) by rigidly aligning all datasets to the series' baseline scan. Having rigidly aligned each dataset to its template, gray matter (GM) was automatically segmented in each image with SPM2.

We then defined three regions of interest (ROIs) in the template images (see Fig. 2) and determined the GM volume as the sum of GM density within these ROIs. We chose large box-shaped ROIs that stretch across the complete slice stack in order to minimize effects resulting from remaining imperfections of the rigid registration.
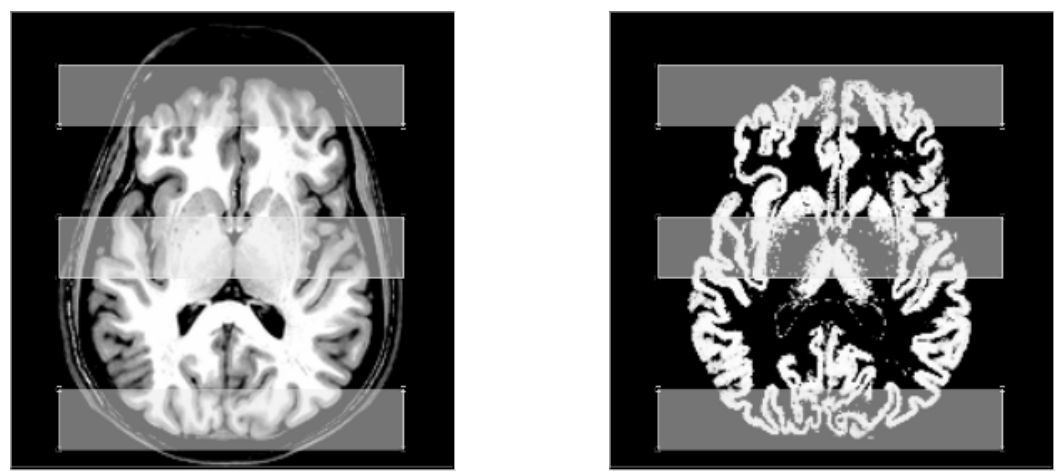

Fig. 2. ROI locations (anterior, medial, posterior) in the Avanto template image (left), and in a GM segmentation image (right)

\section{Results}

\subsection{Validation}

Whereas the precision of the proposed phantom evaluation method is limited by noise and partial volume effects, the reproducibility of the detection of the ellipsoids' center points is additionally limited by temporal drifts in the scanners' resonance frequency during data acquisition and in between two scans. This error in frequency encoding results in a slightly varying discretization scheme in readout direction even within one scanning process. For two consecutive scans this effect looks similar to a slight shift in readout direction in image space. Practical test-retest experiments consisting of evaluations of datasets that were acquired directly one after the other with identical protocols without repositioning resulted in maximal relative positional differences of less than $0.15 \mathrm{~mm}$ for the Avanto, and less than $0.11 \mathrm{~mm}$ for the Trio in a subvolume relevant for brain morphometry (spherical subvolume around the isocenter with a radius of $100 \mathrm{~mm}$ ). The corresponding numbers for relative volume differences are 0.35 $\%$ respectively $0.26 \%$ (relative to the volume of a standard cell in $G 0$ of $7600 \mathrm{~mm}^{3}$ ). The slightly better results for the Trio most likely are a result of a higher signal-tonoise ratio at $3 \mathrm{~T}$. 
Analogous test-retest experiments for validation of the head image evaluation method resulted in the following volumetric changes for the Avanto: anterior ROI $0.50 \%$, medial ROI $0.16 \%$, and posterior ROI $0.35 \%$. The results for the Trio are: anterior ROI $0.25 \%$, medial ROI $0.39 \%$, and posterior ROI $0.12 \%$. Note that the test-retest datasets, too, were rigidly aligned to the template datasets before segmentation.

\subsection{Repositioning Influences}

An important influence on the long-term study's results were expected to be slight differences in positioning. Due to the nonlinear character of the distortion field, a different position of an unaltered object in the scanner is accompanied by relative morphological changes in image space. To get insight into the magnitude of relative geometrical distortions that are purely caused by minor errors in repositioning, the outlined evaluation was performed on two datasets, that, again, were acquired directly one after the other with a slight difference in the phantom's position in the scanner (rotation less than $5^{\circ}$, translation less than $2 \mathrm{~mm}$, new selection of field of view, new shim). These changes produced positional deviations of up to $0.6 \mathrm{~mm}$ (for both scanners), and relative volumetric changes of up to $1.2 \%$ for the Avanto and $1.8 \%$ for the Trio. Relative morphological changes were smaller for the Avanto due to the smaller spatial gradients of the absolute distortion fields (see Sect. 3.3).

Again, analogous repositioning experiments were carried out with the human volunteer, resulting in the following volumetric changes for the Avanto: anterior ROI $0.78 \%$, medial ROI $0.57 \%$, and posterior ROI $1.32 \%$. The corresponding numbers for the Trio are: anterior ROI $0.95 \%$, medial ROI $0.43 \%$, and posterior ROI $0.16 \%$.

\subsection{Long-Term Absolute and Relative Distortions}

Absolute positional and volumetric distortions from the phantom's reference model G0 were computed for the complete data collection for both scanners. It turned out that absolute geometrical distortions are smaller for the Avanto than for the Trio. As expected, geometrical distortions are depending on the object's distance to the magnet's isocenter. In summary, the maximum errors obtained were $2.0 \mathrm{~mm}$ positional deviation and $-3.9 \%$ volume mismatch for the Avanto, and $4.8 \mathrm{~mm}$ respectively $-16.5 \%$ for the Trio within a volume relevant for brain morphometry (spherical subvolume around the isocenter with a radius of $100 \mathrm{~mm}$ ). The difference between the scanners is mainly a result of different gradient coils being integrated in them.

Relative positional and volumetric changes were determined by comparing positions of corresponding spheres, and volumes of corresponding cells with each other rather than against their reference values in $G 0$. Not surprisingly, the magnitude of the distortions respectively volume changes is smaller for the relative than for the absolute evaluations (see Fig. 3 and Fig. 4).

In detail, the maximum relative morphological changes are: $1.0 \mathrm{~mm}$ positional, and $2.0 \%$ volumetric change (relative to a standard cell in $G 0$ of $7600 \mathrm{~mm}^{3}$ ) for the 
Avanto, and $0.6 \mathrm{~mm}$ and $2.0 \%$ for the Trio. The slightly better results for the Trio most likely are a result of higher reproducibility in phantom positioning.
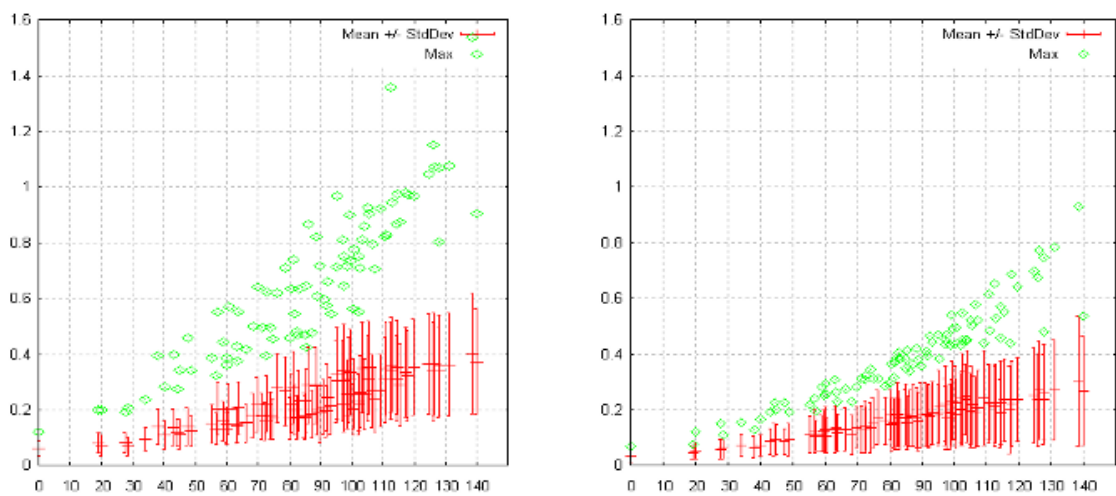

Fig. 3. Distribution of relative positional differences (in $\mathrm{mm}$ ) of detected spheres in dependence of their distance (in $\mathrm{mm}$ ) to the isocenter: Avanto (left), Trio (right).
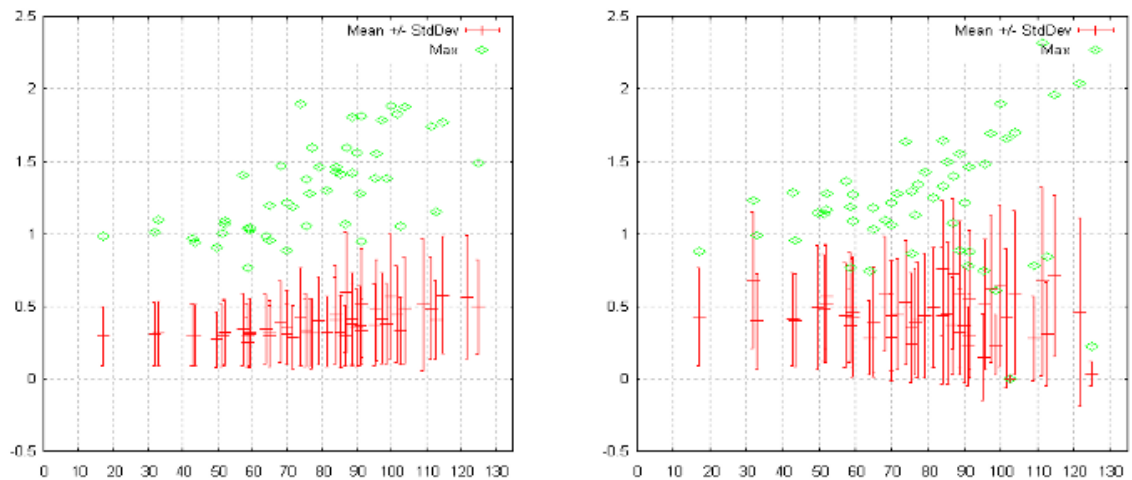

Fig. 4. Distribution of relative volume change (in \% of a standard cell's volume in $G 0$ of 7600 $\mathrm{mm}^{3}$ ) in dependence of the cells' distances (in $\mathrm{mm}$ ) to the isocenter: Avanto (left), Trio (right)

The results of the head image evaluation are given in table 1.

Table 1. Results of head image evaluation: long-term variability in local GM volumetry estimated by range divided by mean (given in brackets)

\begin{tabular}{lcc}
\hline & \multicolumn{1}{c}{ Avanto } & Trio \\
\hline anterior ROI & $2.88 \%\left(\right.$ of $\left.40773.8 \mathrm{~mm}^{3}\right)$ & $4.74 \%\left(\right.$ of $\left.29693.4 \mathrm{~mm}^{3}\right)$ \\
medial ROI & $2.69 \%\left(\right.$ of $\left.187359.2 \mathrm{~mm}^{3}\right)$ & $2.60 \%\left(\right.$ of $\left.184959.6 \mathrm{~mm}^{3}\right)$ \\
posterior ROI & $5.24 \%\left(\right.$ of $\left.68907.8 \mathrm{~mm}^{3}\right)$ & $4.78 \%\left(\right.$ of $\left.86514.3 \mathrm{~mm}^{3}\right)$ \\
\hline
\end{tabular}




\section{Conclusions}

In this paper, the crucial long-term reproducibility of geometrical distortions in MRI was examined for the first time on a local basis. We quantified both practical limitations in serial MRI based morphometry by analyzing head image series of a healthy volunteer acquired over a 6-months period of time on identical hardware, as well as purely acquisition-related limitations by analyzing analogous phantom data series.

As a result of the high reproducibility of our phantom evaluation method $(0.15 \mathrm{~mm}$ maximal relative positional difference and $0.35 \%$ maximal volume difference including effects caused by the scanners' resonance frequency drift), the results obtained from our analyses can be considered a precise quantification of limitations imposed on serial MRI based morphometry by imperfections in the data acquisition process and by patient repositioning errors. To our understanding, the analysis of these effects is an indispensable prerequisite for a profound validation of image processing methods in the field of morphometry.

In detail, the Avanto and the Trio proved to be equally suitable for serial morphometric studies, as their long-term reproducibility of geometrical distortions was equally high: The maximal relative morphological changes were $1.0 \mathrm{~mm}$ positional and $2.0 \%$ volumetric deviation on a $7600 \mathrm{~mm}^{3}$ cuboid. Any morphological changes detected in longitudinal studies carried out with these scanner types that are not larger than the given values have to be interpreted as not significant.

The results of the phantom experiments are well reflected in the evaluations of the head images: Especially cortical areas that are relatively far away from the magnet's isocenter are affected by geometrical distortions. As our volunteer was positioned with great care, and since he was mentally and physically fit and very cooperative, the numbers given in table 1 (up to $5.24 \%$ insecurity in local gray matter volume determination) have to be considered a realistic estimation of serial reproducibility in stateof-the-art MRI based morphometry of cortical areas in terms of a lower limit.

\section{References}

1. Thompson. P.M. et al.: Mapping Cortical Change in Alzheimer's Disease, Brain Development, and Schizophrenia; Neuroimage, 23 Suppl 1, S2-18, 2004

2. Fischl, B. et al. : Whole Brain Segmentation: Automated Labeling of Neuroanatomical Structures in the Human Brain; Neuron, 33, 341-355, 2002

3. Ashburner, J.T., Friston K.J.: Voxel-Based Morphometry - The Methods; NeuroImage, 11, 805-821, 2000

4. Jovicich, J. et al.: Reliability in Multi-Site Structural MRI Studies: Effects of Gradient Nonlinearity Correction on Volume and Displacement of Brain Subcortical Structures; Human Brain Mapping, Budapest, 2004

5. Jovicich, J. et al.: Multi-site Structural MRI Studies: An Evaluation of Image Distortions and Image Intensity Reproducibility; ISMRM 2004

6. Haacke, E.M.: Magnetic Resonance Imaging - Physical Principles and Sequence Design; Wiley, 1999

7. Otsu, N.: A Threshold Selection Method from Grey-Level Histograms; IEEE Transactions on System, Man, and Cybernetics, 9, 62-66, 1979 